\title{
Application of Virtualization Technology in Computer Experiments
}

\author{
Huiran Zhao ${ }^{1, \text { a }}$, Lei Shi ${ }^{2, b}$, Shujian $\mathrm{Zhu}^{3, \mathrm{c}}$ \\ ${ }^{1,2,3}$ School of Computer Engineering,City Institude,Dalian University of Technology,Dalian 116600 \\ China \\ àzhr2006@dlut.edu.cn, bshilei@dlut.edu.cn, ${ }^{c} 1669452948 @$ qq.com
}

Keywords: Virtualization technology, Virtualization.

\begin{abstract}
The main reason for applying virtualization technology in computer experimental teaching is to highlight the common participation and mutual cooperation between teachers and students, and combine the basic concept of "student centered". This is not only the need of teaching practice, but also the need of teaching reform. It has practical significance and practical value for the training of students' practical ability and practical ability and the improvement of their operation skills. Through the research, summed up the characteristics and advantages of existing mainstream virtualization products, to find suitable for laboratory use types of software, and ultimately determine the selection of software; design a specific scheme of virtual experimental computer courses and the implementation of the content and experimental teaching in the implementation of quality education goals and evaluation plan; learning by changing and improve the students' practical ability and practice ability, the teaching practice of computer class has more practical significance.
\end{abstract}

\section{The background and significance of virtualization technology}

Virtualization technology is a branch of computer science, was created in the $60 \mathrm{~s}$ and $70 \mathrm{~s}$ of the last century, based on the partition technology for IBM mainframes. Since the ninety s, with the development of computer science and the change of market demand, virtualization technology brings advantages such as cost savings, improve security gradually get attention, the server merge, network security, data protection calculation, high performance computing and credible, and other fields have a large number of applications, is a hot research topic in the field of computer science in the world.

Virtualization in computer applications usually refers to the operation of computer components on virtual machines rather than real machines. The virtual server is a physical server by way of software in the server virtual a virtual server, the virtual server is called virtual machine, each virtual machine can have their own CPU, memory, hard disk, network card and other hardware, at the same time between virtual machines are in an independent manner, each other no interference. Different types of operating systems and applications can be installed on virtual machines to meet different IT hardware requirements.

Server virtualization can ease the shortage of resources and the shortage of resources in university labs from two aspects of technology and management. It also provides a feasible way for constructing new teaching experimental platform. The experimental teaching platform based on virtualization technology not only saves equipment outlay, but also meets the diversity, complexity and experimental demand of computer experiment teaching. In order not to increase hardware 
investment, it makes full use of the existing experimental equipment potential, improve the utilization efficiency of the existing equipment, increasing the number of students the opportunity to practice with virtualization technology is improving, the concept of virtualization and virtualization technology solutions will in the construction of University experimental teaching platform in the play a more active role.

Computer labs in universities emphasize the cultivation of students' skills rather than their research work. In order to really meet the needs of students' skills training, we need to solve the sufficient number of equipment first. If each student has an independent server, at least two computers, routers, switches, firewalls and other equipment. Second, different manufacturers and models of equipment, functions are also different. Virtualization technology can solve the two problems mentioned above to some extent, the use of virtualization technology, a common computer laboratory can be built successfully complete, advanced equipment and specialized laboratories, each student can according to the need of virtual computer, network equipment, virtual devices do not need to maintain, will not be damaged, even students can be a virtual laboratory equipment of the home, in the bedroom can continue to learn. Whether for schools or individuals, you can create almost zero cost experimental environment according to the content of the textbooks.

\section{Analysis of the main problems faced by computer resources in Colleges and Universities}

The culture teaching philosophy emphasizes students' practical ability and innovative ability, training goal of computer specialty is more emphasis on the "in the computer teaching focus on practical teaching" and emphasizing practical ability. This is a significant change in the original classroom curriculum for teaching in the laboratory, such as program design course "original classroom teaching and computer is two links", instead of "students learn while training" in the laboratory, the assessment test practical courses, this course makes the utilization of laboratory a substantial increase. These reasons often cause overloading of computer labs, and laboratory administrators are difficult to maintain laboratory due to heavy load。

The student's own computer equipment is less efficient in learning. In computer science, almost $100 \%$ of all sophomores have their own computers. Although most students can use their computers in their studies, these computers have not been fully utilized. In the course of the class, these computers are idle, and a lot of students with poor self-control are learning tools as entertainment tools, and their computers are not really used for learning.

There is a contradiction between basic machine and professional machine. It is easy to think that the computer resources used in the teaching of basic courses and the teaching of nuclear courses should be shared, but it is very difficult to do in reality. The number of students for dozens of experimental courses, must ensure that all software after careful installation and debugging, the software needs to have good compatibility, or prone to crashes, blue screen, do not have access to the Internet, can not be applied to such problems. Once this problem occurs, it will become a serious teaching accident, which is also the most worrying for the class teacher. The installation, use the computer professional course to uninstall or update software is It is quite common for things, so it is very prone to the problem, although the teaching laboratory for the installation of computer protection card, but the computer restart after all recovery, will bring great inconvenience to the teaching of professional courses, because some students installed software is need to restart the application of computer to normal.

The rapid elimination of equipment brings costly expenses. By contrast, a single computer device is not expensive, but because of the large amount and the 5 - to 6 - year culling cycle, it is expensive to have a computer lab that meets all of the teaching requirements. How to extend the 
useful life of these equipment and make use of a lot of free computer resources may be a problem that must be solved in the construction of a conservation minded society.

The choice of experiment content is restricted by hardware condition. At present, in order to simplify the management of university computer lab, the password is set to computer equipment BIOS, which makes students unable to exercise BIOS settings; motherboard with hard disk protection card, which makes students unable to install new software; using laboratory management software shielded the control panel in the operating system, which makes the students' experimental settings to practice operating system, computer maintenance experiments, such as a hard disk partition, format, operating system installation, optimization, driver installation and other modifications of experiment on the disk, these experiments can enable students to personally finish. These directly affect the students' hands-on ability, so that the computer students lack the basic computer maintenance capabilities. For example, the experiment of computer network course, such as the setting up of LAN, the configuration and management of network, the configuration of IIS, FTP and Mail, the configuration of various network servers, the configuration of switches and routes, etc.. The network experiment need to use multiple computers and network equipment, but due to the limited experimental conditions, students after studying the related courses of theoretical knowledge can not be carried out experiments that lead to students unable to solve practical problems encountered in the future work.

In the process of network equipment debugging experiment, colleges and universities need to build new specialized laboratories, and purchase a considerable number of switches, routers, firewalls and other equipment to meet the experimental needs of students. However, the network equipment has many problems, such as many types, expensive and fast updating. In addition, due to class time and place restrictions, students can only use the equipment within the time stipulated in the school. The laboratory can not be opened all day for safety reasons. Because of the lack of laboratory opening time, it is impossible to finish the experiment on time. To solve this kind of problem, we can make use of simulation software to network equipment virtualization, such software can run on the ordinary computer, used to simulate the mainstream switches, routers and other network devices.

\section{Application of virtualization technology in experiment of computer courses}

Application of "network security foundation" course. In this course the safety requirements are relatively high, add the contents of a large number of experiments using the virtual machine to complete the experiment, for example, to build a VPN server, if the server is using real hard to achieve a computer, but also easily lead to a system crash situation often occurs in the real VPN configuration errors in students the server, resulting in equipment to the normal application, virtualization is the perfect solution to this problem. Each student can build and configure the server through the virtual machine, even if the experiment fails, it will not affect the normal operation of other students' computer. Serious problems in the process of the server configuration, if it can not be resolved, but also by a simple reduction to restart, re configuration can, this method is simple and convenient and can not damage the hardware, everyone can personally solve the problem.

Application in the "operating system" course. In the course of operating system experiment, if you are using a real computer is impossible for students to set the related hardware, such as a hard disk partition, format, operating system installation, optimization, driver installation and other modifications of experiment on the disk, which can let the students to complete, which directly affects the ability of students culture, make computer students lack basic computer maintenance ability. After the use of virtualization technology, the experimental content has been greatly 
enriched, the virtual device can be arbitrarily set, without fear of damage to the laboratory hardware equipment.

The application of "Internet technology should" course. The main part is to adjust the part of virtual private network. The experiment can simulate the remote access virtual network (Access-VPN) through the virtual machine. The method is to install the Windows XP operating system in the student's computer, it is used to simulate the remote client; a virtual machine to install in the Windows XP operating system, Windows Server 2003 server installed in the virtual machine, and configure a FTP server in the server for remote users access. Establish a VPN connection between the Windows XP and the Windows Server server to simulate a VPN tunnel, so that remote users access the intranet FTP server as if it were local. In this way, each student can set up a set of experimental environment, each student can independently complete the content of the experiment, and solve the problems in the experiment independently.

The application of "Linux system management" course. After several rounds of teaching adjustment and testing, the current Linux system management course experimental environment has been fully implemented by the virtual machine software. Fully able to configure all types of servers in the Linux operating system through virtual machine software.

Application in the course of computer network. Is mainly developed by American CISCO company equipment simulation software Cisco Packet Tracer, the software developed by Cisco network products, simple installation, convenient operation, friendly interface, fully functional, widely recognized, suitable for primary students to use. We have already developed a lot of experiment or practice teaching task or project with this software, and have carried on the actual application, and obtained the good result. The courses used in these virtual experiment projects include computer network experiment, network engineering design, LAN practice and campus network practice. In the computer network experiment courses, we have written the notes "related to computer network selection experiments and exercises", the book describes the basic usage of Packet Tracer device simulation software Cisco, and developed some experimental tasks.

in the business website design and management of the application. Mainly reflected in the use of "online classroom" virtualization, "online classroom" module to achieve the function of online lectures. Students can not only watch the teaching video, but also in the course of lectures, the system will be at a certain point in time to see the video content of the relevant questions. If the answer is a failure, you can't continue to watch the instructional video. You need to start looking for the right answer again. If teachers in the curriculum in time, so when students play video courses and achieve the point in time, will pause, and prompted to answer the question, if the question is answered correctly, then you can continue to play the video, if the answer is wrong, then the system will be returned to the user player time point or a video head. The video player's progress bar cannot be dragged, so students can't change the playback schedule or skip the time points.

\section{Advantages of virtualization technology in computer practice teaching}

Teaching superiority. Virtualization technology provides an effective means for computer practice teaching, and the virtual machine experimental environment simulates the real environment well. The experiments performed on virtual machines are equivalent to the experimental results done on real machines, which are in full accordance with the teaching requirements. In the virtual environment, students can boldly carry out a variety of experimental operations, without worrying about the destruction of the host system, virtual experiment environment can increase students' practice, and strengthen students' practical skills. The construction of virtual experiment environment based on virtual machine technology, to a certain extent, it can not only alleviate the 
current shortage of experimental equipment, but also improve the largely experimental equipment safety and utilization, reduce the difficulty of laboratory management.

Cost advantage. The application of virtualization technology can not only greatly reduce the cost, but also effectively reduce the energy consumption in the experimental process, and the efficiency and quality of the computer experimental teaching can be greatly improved. At the time, resources and cost of three heavy pressure, how to make higher education quality and efficiency of computer experimental teaching more efficiently, using virtualization technology VMware, build a suitable for all applications of the PC virtual machine environment, reduce the amount of hardware required by the current in order to improve the utilization rate of resources. To improve the teaching effect, improve teaching environment. A lot of computer servers and the existing energy consumption is relatively large, and the virtualization technology of type VMware with memory management and advanced resource features, can achieve the integration rate of up to 15:1 or even higher, hardware utilization increased to $85 \%$, and some do not use intelligent physical servers close, minimize the consumption of energy is not reduce the functional integrity and reliability.

Management advantage. The use of virtualization technology, combined with the particularity of students in Colleges and universities, the construction of a reasonable and perfect computer experimental teaching system, and promote the smooth progress of teaching and research work. The good experiment teaching of computer in Colleges and universities is not only to have several experimental teaching courses, but also to establish a good teaching system, which is easy to manage.

\section{Future research ideas}

The progress of virtualization technology has promoted the teaching of computer experiments, and can better improve students' practical ability. We should pay full attention to an important role of virtual technology in computer experiment teaching, and rationally use, continuous learning, the only way to better improve the problems in computer experiment teaching, improve the quality of computer experiment teaching.

\section{References}

[1] Chu Jun. The Metaphor Interpretation of Narrative News Headline from the Perspective of Narrative Approach[J].Foreign Language World,2012,01:90-95.

[2] Zhang Yan. The Image Presentation and Aesthetics Turn of Data News[J]. Editors' Friend,2015,03:85-88.

[3] Li Yan, Li Saike. Tell a Good Story? How Data Journalism Inherits and Transforms Traditional Journalism[J]. Journal of Zhejiang University (Humanities and Social Sciences), 2015, 06:106-128.

[4] Li Xin.Schema Understanding: Cognitive Mechanism and Narrative Meaning of Minority Journalism[J]. Contemporary Communication, 2016,04:57-60.

[5] Yang Guang, Gou Zhongshi. News Content, Understanding and Memory: A Mental Model for Comprehending the Coverage of Controversial Issues[J]. Journalism \& Communication, 2016, $11: 35-50+126-127$. 